\title{
A Starting Method for Solving Nonlinear Volterra Integral Equations
}

\author{
By J. T. Day
}

Abstract. In this paper a fifth order starting method is given for Volterra equations of the form $y(t)=f(t)+\int_{x_{0}}^{t} l i(t, s, y(s)) d s$. Computational examples are given for the method as a starting method for the Gregory-Newton method.

1. Introduction. In this paper we shall consider an $O\left(h^{5}\right)$ starting method for the numerical solution of the nonlinear Volterra integral equation

$$
y(t)=f(t)+\int_{x_{0}}^{t} k(t, s, y(s)) d s .
$$

After stating our algorithm we shall discuss its deriviation and consider some computational examples. In our computational examples we shall consider our method as a starting method for the Gregory-Newton method. The Gregory-Newton method in this context has been discussed by Fox and Goodwin [2], Noble [8], and Todd [11].

2. The Algorithm. The self-starting method described here advances the solution from $x_{0}$ to $x_{0}+h, x_{0}+h$ to $x_{0}+2 h, \cdots, x_{0}+5 h$ to $x_{0}+6 h$. To advance from $x_{0}$ to $x_{0}+h$ we compute

$$
\begin{aligned}
\hat{y}_{1 / 3}=f\left(x_{0}+\frac{h}{3}\right)+\frac{h}{3} k\left(x_{0}+\frac{h}{3}, x_{0}, y_{0}\right), \\
y_{1 / 3}=f\left(x_{0}+\frac{h}{3}\right)+\frac{h}{6}\left[k\left(x_{0}+\frac{h}{3}, x_{0}, y_{0}\right)+k\left(x_{0}+\frac{h}{3}, x_{0}+\frac{h}{3}, \hat{y}_{1 / 3}\right)\right], \\
\hat{y}_{2 / 3}=f\left(x_{0}+\frac{2 h}{3}\right)+\frac{2 h}{3} k\left(x_{0}+\frac{2 h}{3}, x_{0}+\frac{h}{3}, y_{1 / 3}\right), \\
\hat{y}_{1 / 2}=f\left(x_{0}+\frac{h}{2}\right)+\frac{h}{8}\left[k\left(x_{0}+\frac{h}{2}, x_{0}, y_{0}\right)+3 k\left(x_{0}+\frac{h}{2}, x_{0}+\frac{h}{3}, y_{1 / 3}\right)\right], \\
\hat{y}_{1}=f\left(x_{0}+h\right)+\frac{h}{4}\left[k\left(x_{0}+h, x_{0}, y_{0}\right)+3 k\left(x_{0}+h, x_{0}+\frac{2 h}{3}, \hat{y}_{2 / 3}\right)\right], \\
y_{1}=f\left(x_{0}+h\right)+\frac{h}{6}\left[k\left(x_{0}+h, x_{0}, y_{0}\right)\right. \\
\left.+4 k\left(x_{0}+h, x_{0}+\frac{h}{2}, \hat{y}_{1 / 2}\right)+k\left(x_{0}+h, x_{0}+h, \hat{y}_{1}\right)\right] .
\end{aligned}
$$

To advance from $x_{0}+h$ to $x_{0}+2 h$ we compute

$$
\hat{y}_{3 / 2}=f\left(x_{0}+\frac{3 h}{2}\right)+\frac{3 h}{4}\left[k\left(x_{0}+\frac{3 h}{2}, x_{0}+\frac{h}{2}, \hat{y}_{1 / 2}\right)\right.
$$

Received March 7, 1966. Revised August 25, 1966.

$$
\left.+k\left(x_{0}+\frac{3 h}{2}, x_{0}+h, y_{1}\right)\right],
$$




$$
\begin{array}{r}
y_{3 / 2}=f\left(x_{0}+\frac{3 h}{2}\right)+\frac{3 h}{16}\left[k\left(x_{0}+\frac{3 h}{2}, x_{0}, y_{0}\right)+3 k\left(x_{0}+\frac{3 h}{2}, x_{0}+\frac{h}{2}, \hat{y}_{1 / 2}\right)\right. \\
\left.+3 k\left(x_{0}+\frac{3 h}{2}, x_{0}+h, y_{1}\right)+k\left(x_{0}+\frac{3 h}{2}, x_{0}+\frac{3 h}{2}, \hat{y}_{3 / 2}\right)\right], \\
\hat{y}_{2}=f\left(x_{0}+2 h\right)+\frac{2 h}{3}\left[k\left(x_{0}+2 h, x_{0}+\frac{h}{2}, \hat{y}_{1 / 2}\right) \cdot 2\right. \\
\left.-k\left(x_{0}+2 h, x_{0}+h, y_{1}\right)+2 k\left(x_{0}+2 h, x_{0}+\frac{3 h}{2}, y_{3 / 2}\right)\right], \\
y_{2}=f\left(x_{0}+2 h\right)+\frac{h}{6}\left[k\left(x_{0}+2 h, x_{0}, y_{0}\right)+4 k\left(x_{0}+h 2, x_{1 / 2}, \hat{y}_{1 / 2}\right)\right. \\
\left.+2 k\left(x_{2}, x_{1}, y_{1}\right)+4 k\left(x_{2}, x_{3 / 2}, y_{3 / 2}\right)+k\left(x_{2}, x_{2}, \hat{y}_{2}\right)\right] .
\end{array}
$$

To advance from $x_{0}+2 h$ to $x_{0}+3 h$ we compute

$$
\begin{aligned}
\hat{y}_{5 / 2}= & f\left(x_{0}+\frac{5 h}{2}\right)+\frac{5 h}{48}\left[11 k\left(x_{0}+\frac{5 h}{2}, x_{0}+\frac{h}{2}, \hat{y}_{1 / 2}\right)\right. \\
+ & k\left(x_{0}+\frac{5 h}{2}, x_{0}+h, y_{1}\right)+k\left(x_{0}+\frac{5 h}{2}, x_{0}+\frac{3 h}{2}, y_{3 / 2}\right) \\
& \left.+11 k\left(x_{0}+\frac{5 h}{2}, x_{0}+2 h, y_{2}\right)\right], \\
y_{5 / 2}=f & \left(x_{0}+\frac{5 h}{2}\right)+\frac{5 h}{576}\left[19 k\left(x_{0}+\frac{5 h}{2}, x_{0}, y_{0}\right)\right. \\
& +75 k\left(x_{0}+\frac{5 h}{2}, x_{1 / 2}, \hat{y}_{1 / 2}\right)+50 k\left(x_{0}+\frac{5 h}{2}, x_{1}, y_{1}\right) \\
+ & \left.50 k\left(x_{0}+\frac{5 h}{2}, x_{3 / 2}, y_{3 / 2}\right)+75 k\left(x_{5 / 2}, x_{2}, y_{2}\right)+19 k\left(x_{5 / 2}, x_{5 / 2}, \hat{y}_{5 / 2}\right)\right] \\
\hat{y}_{3}= & f\left(x_{0}+3 h\right)+\frac{3 h}{20}\left[11 k\left(x_{3}, x_{1 / 2}, \hat{y}_{1 / 2}\right)-14 k\left(x_{3}, x_{1}, y_{1}\right)\right. \\
& \left.\quad+26 k\left(x_{3}, x_{3 / 2}, y_{3 / 2}\right)-14 k\left(x_{3}, x_{2}, y_{2}\right)+11 k\left(x_{3}, x_{5 / 2}, y_{5 / 2}\right)\right] \\
y_{3}= & f\left(x_{0}+3 h\right)+\frac{h}{6}\left[k\left(x_{3}, x_{0}, y_{0}\right)+4 k\left(x_{3}, x_{1 / 2}, \hat{y}_{1 / 2}\right)+2 k\left(x_{3}, x_{1}, y_{1}\right)\right. \\
+ & \left.4 k\left(x_{3}, x_{3 / 2}, y_{3 / 2}\right)+2 k\left(x_{3}, x_{2}, y_{2}\right)+4 k\left(x_{3}, x_{5 / 2}, y_{5 / 2}\right)+k\left(x_{3}, x_{3}, \hat{y}_{3}\right)\right] .
\end{aligned}
$$

To advance from $x_{0}+3 h$ to $x_{0}+4 h$ we compute

(16) $\hat{y}_{4}=f\left(x_{0}+4 h\right)+\frac{4 h}{3}\left[2 k\left(x_{4}, x_{1}, y_{1}\right)-k\left(x_{4}, x_{2}, y_{2}\right)+2 k\left(x_{4}, x_{3}, y_{3}\right)\right]$,

$$
\begin{aligned}
y_{4}=f\left(x_{0}+4 h\right) & +\frac{4 h}{90}\left[7 k\left(x_{0}+4 h, x_{0}, y_{0}\right)+32 k\left(x_{0}+4 h, x_{0}+h, y_{1}\right)\right. \\
+ & \left.12 k\left(x_{0}+4 h, x_{2}, y_{2}\right)+32 k\left(x_{4}, x_{3}, y_{3}\right)+7 k\left(x_{4}, x_{4}, \hat{y}_{4}\right)\right]
\end{aligned}
$$


To advance from $x_{0}+4 h$ to $x_{0}+5 h$ we compute

$$
\begin{aligned}
& \hat{y}_{5}=f\left(x_{0}+5 h\right)+\frac{5 h}{24}\left[11 k\left(x_{5}, x_{1}, y_{1}\right)+k\left(x_{5}, x_{2}, y_{2}\right)\right.+k\left(x_{5}, x_{3}, y_{3}\right) \\
&\left.+11 k\left(x_{5}, x_{4}, y_{4}\right)\right], \\
& y_{5}=f\left(x_{0}+5 h\right)+\frac{5 h}{288}\left[19 k\left(x_{0}+5 h, x_{0}, y_{0}\right)+75 k\left(x_{0}+5 h, x_{0}+h, y_{1}\right)\right. \\
&\left.+50 k\left(x_{5}, x_{2}, y_{2}\right)+50 k\left(x_{5}, x_{3}, y_{3}\right)+75 k\left(x_{5}, x_{4}, y_{4}\right)+19 k\left(x_{5}, x_{5}, \hat{y}_{5}\right)\right] .
\end{aligned}
$$

To advance from $x_{0}+5 h$ to $x_{0}+6 h$ we compute

$$
\begin{aligned}
& \hat{y}_{6}=f\left(x_{0}+6 h\right)+ \frac{6 h}{20}\left[11 k\left(x_{6}, x_{1}, y_{1}\right)-14 k\left(x_{6}, x_{2}, y_{2}\right)\right. \\
&\left.+26 k\left(x_{6}, x_{3}, y_{3}\right)-14 k\left(x_{6}, x_{4}, y_{4}\right)+11 k\left(x_{6}, x_{5}, y_{6}\right)\right], \\
& y_{6}=f\left(x_{0}+6 h\right)+ \frac{3 h}{10}\left[k\left(x_{6}, x_{0}, y_{0}\right)+5 k\left(x_{6}, x_{1}, y_{1}\right)+k\left(x_{6}, x_{2}, y_{2}\right)\right. \\
&\left.+6 k\left(x_{6}, x_{3}, y_{3}\right)+k\left(x_{6}, x_{4}, y_{4}\right)+5 k\left(x_{6}, x_{5}, y_{5}\right)+k\left(x_{6}, x_{6}, \hat{y}_{6}\right)\right] .
\end{aligned}
$$

3. Derivation of Algorithm. We shall sketch the derivation of the algorithm. Many of the ideas for the algorithm will be found in a paper due to Kuntzmann [5].

If we approximate the integral in (1) by Simpson's rule on the interval $\left[x_{0}, x_{0}+h\right]$ we obtain

$$
\begin{aligned}
y\left(x_{0}+h\right)= & f\left(x_{0}+h\right)+\frac{h}{6}\left[k\left(x_{0}+h, x_{0}, y_{0}\right)\right. \\
& +4 k\left(x_{0}+h, x_{0}+\frac{h}{2}, y\left(x_{0}+\frac{h}{2}\right)\right) \\
& \left.+k\left(x_{0}+h, x_{0}+h, y\left(x_{0}+h\right)\right)\right]-\frac{h^{5}}{2880} k^{\mathbf{I V}}\left(x_{0}+h, \xi, y(\xi)\right) .
\end{aligned}
$$

where $x_{0}<\xi<x_{0}+h$. Here $y\left(x_{0}+h / 2\right)$ and $y\left(x_{0}+h\right)$ are not known in the right side of $(22)$. If we are to use (22) we must obtain accurate approximate values for $y\left(x_{0}+h / 2\right)$ and $y\left(x_{0}+h\right)$. We do this in the following manner. First we note that

$$
\begin{aligned}
y\left(x_{0}+h\right)=f\left(x_{0}+h\right)+ & \frac{h}{4}\left[k\left(x_{0}+h, x_{0}, y_{0}\right)\right. \\
& \left.+3 k\left(x_{0}+h, x_{0}+\frac{2 h}{3}, y\left(x_{0}+\frac{2 h}{3}\right)\right)\right]+O\left(h^{4}\right)
\end{aligned}
$$

is an $O\left(h^{4}\right)$ approximation to $y\left(x_{0}+h\right)$. (This is the Radau two-point rule.) However, here we do not know $y\left(x_{0}+2 h / 3\right)$, but if we could obtain it to $O\left(h^{3}\right)$ then we could use (23). Thus, we attempt to attain an $O\left(h^{3}\right)$ approximation to $y\left(x_{0}+2 h / 3\right)$. This is done by using the midpoint rule

$$
y\left(x_{0}+\frac{2 h}{3}\right)=f\left(x_{0}+\frac{2 h}{3}\right)+\frac{2 h}{3} k\left(x_{0}+\frac{2 h}{3}, x_{0}+\frac{h}{3}, y\left(x_{0}+\frac{h}{3}\right)\right)+O\left(h^{3}\right) .
$$


However here we do not know $y\left(x_{0}+h / 3\right)$ to $O\left(h^{2}\right)$. We obtain it to $O\left(h^{3}\right)$ by using the trapezoidal rule and Taylor's series

$$
\begin{aligned}
y\left(x_{0}+\frac{h}{3}\right)=f\left(x_{0}+\frac{h}{3}\right)+ & \frac{h}{6}\left[k\left(x_{0}+\frac{h}{3}, x_{0}, y_{0}\right)\right. \\
& \left.+k\left(x_{0}+\frac{h}{3}, x_{0}+\frac{h}{3}, y\left(x_{0}+\frac{h}{3}\right)\right)\right]+O\left(h^{3}\right), \\
y\left(x_{0}+\frac{h}{3}\right)= & f\left(x_{0}+\frac{h}{3}\right)+\int_{x_{0}}^{x_{0}+h / 3}\left[k\left(x_{0}+\frac{h}{3}, x_{0}, y_{0}\right)+O(h)\right] d s \\
= & f\left(x_{0}+\frac{h}{3}\right)+\frac{h}{3} k\left(x_{0}+\frac{h}{3}, x_{0}, y_{0}\right)+O\left(h^{2}\right) .
\end{aligned}
$$

Summarizing the above procedure, we have that formula (23) is used to predict a value for $y_{1}$ (Eq. (6)) which is then corrected with (25) (Eq. (7)). Formula (26) is used to predict a value for $y_{1 / 3}$ (Eq. (2)) which is corrected with (25) (Eq. (3)).

The value of $\hat{y}_{1 / 2}$ is obtained by approximating the integral in

$$
y\left(x_{0}+\frac{h}{2}\right)=f\left(x_{0}+\frac{h}{2}\right)+\int_{x_{0}}^{x_{0}+h / 2} k(t, s, y(s)) d s, \quad t=x_{0}+\frac{h}{2},
$$

by the Radau two-point rule, disregarding the truncation error and substituting $y_{1 / 3}$ in for $y\left(x_{0}+h / 3\right)$.

In advancing from $x_{0}+h$ to $x_{0}+2 h$, we first let $x$ equal to $x_{0}+2 h$ in (1) to obtain

$$
y\left(x_{0}+2 h\right)=f\left(x_{0}+2 h\right)+\int_{x_{0}}^{x_{0}+2 h} k\left(x_{0}+2 h, s, y(s)\right) d s .
$$

This integral could be evaluated by Simpson's rule if we knew accurate approximate values for $y_{3 / 2}$ and $y_{2}$. We obtain approximate values for $y_{3 / 2}$ by first using the open Newton-Cotes formula

$$
\begin{aligned}
\hat{y}\left(x_{0}+\frac{3 h}{2}\right)=f\left(x_{0}+\frac{3 h}{2}\right)+\frac{3 h}{4}[k & \left(x_{0}+\frac{3 h}{2}, x_{0}+\frac{h}{2}, y_{1 / 2}\right) \\
& \left.+k\left(x_{0}+\frac{3 h}{2}, x_{0}+h, y_{1}\right)\right]+O\left(h^{3}\right)
\end{aligned}
$$

and substituting this value into Simpson's three-eighths' rule on $\left[x_{0}, x_{0}+3 h / 2\right]$

$$
\begin{aligned}
y\left(x_{0}+\frac{3 h}{2}\right)=f\left(x_{0}+\frac{3 h}{2}\right)+\frac{3 h}{16} & {\left[k\left(x_{3 / 2}, x_{0}, y_{0}\right)+3 k\left(x_{3 / 2}, x_{1 / 2}, y_{1 / 2}\right)\right.} \\
& \left.+3 k\left(x_{3 / 2}, x_{1}, y_{1}\right)+k\left(x_{3 / 2}, x_{3 / 2}, \hat{y}_{3 / 2}\right)\right]+O\left(h^{4}\right) .
\end{aligned}
$$

An accurate value for $y\left(x_{0}+2 h\right)$ is obtained by using the Newton-Cotes open formula

$$
\begin{aligned}
y\left(x_{0}+2 h\right)=f\left(x_{0}+2 h\right)+\frac{2 h}{3}\left[2 k\left(x_{2}, x_{1 / 2}, y_{1 / 2}\right)-\right. & k\left(x_{0}+2 h, x_{1}, y_{1}\right) \\
& \left.+2 k\left(x_{2}, x_{3 / 2}, y_{3 / 2}\right)\right]+O\left(h^{5}\right) .
\end{aligned}
$$


and substituting this result into Simpson's rule

$$
\begin{array}{r}
y\left(x_{0}+2 h\right)=f\left(x_{0}+2 h\right)+\frac{h}{6}\left[k\left(x_{2}, x_{0}, y_{0}\right)+4 k\left(x_{2}, x_{1 / 2}, y_{1 / 2}\right)+2 k\left(x_{2}, x_{1}, y_{1}\right)\right. \\
\left.+4 k\left(x_{2}, x_{3 / 2}, y_{3 / 2}\right)+k\left(x_{2}, x_{2}, y_{2}\right)\right]+O\left(h^{5}\right) .
\end{array}
$$

To advance from $x_{0}+2 h$ to $x_{0}+3 h$ we could again use Simpson's rule if we knew accurate approximate values for $y_{5 / 2}$ and $y_{3}$. We proceed as follows. Use the open Newton-Cotes formula

$$
\begin{aligned}
\hat{y}_{5 / 2}=f\left(x_{0}+\frac{5 h}{2}\right)+\frac{5 h}{48} & {\left[11 k\left(x_{0}+\frac{5 h}{2}, x_{1 / 2}, y_{1 / 2}\right)+k\left(x_{0}+\frac{5 h}{2}, x_{1}, y_{1}\right)\right.} \\
& \left.+k\left(x_{0}+\frac{5 h}{2}, x_{3 / 2}, y_{3 / 2}\right)+11 k\left(x_{5 / 2}, x_{2}, y_{2}\right)\right]+O\left(h^{5}\right)
\end{aligned}
$$

along with the closed Newton-Cotes formula

$$
\begin{aligned}
y_{5 / 2}=f\left(x_{0}+\frac{5 h}{2}\right)+ & \frac{5 h}{576}\left[19 k\left(x_{5 / 2}, x_{0}, y_{0}\right)+75 k\left(x_{5 / 2}, x_{1 / 2}, y_{1 / 2}\right)\right. \\
& +50 k\left(x_{5 / 2}, x_{1}, y_{1}\right)+50 k\left(x_{5 / 2}, x_{3 / 2}, y_{3 / 2}\right) \\
& \left.+75 k\left(x_{5 / 2}, x_{2}, y_{2}\right)+19 k\left(x_{5 / 2}, x_{5 / 2}, \hat{y}_{5 / 2}\right)\right]+O\left(h^{5}\right) .
\end{aligned}
$$

To obtain an approximate value for $y$ at $x_{3}$ we use the open Newton-Cotes formula

$$
\begin{aligned}
y_{3}=f\left(x_{0}+\right. & 3 h)+\frac{3 h}{20}\left[11 k\left(x_{0}+3 h, x_{1 / 2}, y_{1 / 2}\right)-14 k\left(x_{3}, x_{1}, y_{1}\right)\right. \\
& \left.+26 k\left(x_{3}, x_{3 / 2}, y_{3 / 2}\right)-14 k\left(x_{3}, x_{2}, y_{2}\right)+11 k\left(x_{3}, x_{5 / 2}, y_{5 / 2}\right)\right]+O\left(h^{6}\right)
\end{aligned}
$$

together with Simpson's rule

$$
\begin{aligned}
y\left(x_{0}+3 h\right)= & f\left(x_{0}+3 h\right)+\frac{h}{6}\left[k\left(x_{0}+3 h, x_{0}, y_{0}\right)+4 k\left(x_{3}, x_{1 / 2}, y_{1 / 2}\right)\right. \\
+ & 2 k\left(x_{3}, x_{1}, y_{1}\right)+4 k\left(x_{3}, x_{3 / 2}, y_{3 / 2}\right)+2 k\left(x_{3}, x_{2}, y_{2}\right) \\
& \left.\quad+4 k\left(x_{3}, x_{5 / 2}, y_{5 / 2}\right)+k\left(x_{3}, x_{3}, y_{3}\right)\right]+O\left(h^{5}\right) .
\end{aligned}
$$

It should be noted that the predictor is of higher order than the corrector here. To advance from $x_{0}+3 h$ to $x_{0}+4 h$ we approximate the integral in

$$
y\left(x_{0}+4 h\right)=f\left(x_{0}+4 h\right)+\int_{x_{0}}^{x_{0}+4 h} k\left(x_{0}+4 h, s, y(s)\right) d s
$$

by the Newton-Cotes formula

$$
\begin{array}{r}
y\left(x_{0}+4 h\right)=f\left(x_{0}+4 h\right)+\frac{4 h}{90}\left[7 k\left(x_{4}, x_{0}, y_{0}\right)+32 k\left(x_{4}, x_{1}, y_{1}\right)+12 k\left(x_{4}, x_{2}, y_{2}\right)\right. \\
\left.+32 k\left(x_{4}, x_{3}, y_{3}\right)+7 k\left(x_{4}, x_{4}, y_{4}\right)\right]+O\left(h^{7}\right) .
\end{array}
$$


Here $y_{4}$ is obtained from the open Newton-Cotes formula

$$
\begin{aligned}
y\left(x_{0}+4 h\right)=f\left(x_{0}+4 h\right)+\frac{4 h}{3}\left[2 k\left(x_{4}, x_{1}, y_{1}\right)-k\left(x_{4}, x_{2}, y_{2}\right)\right. & \\
& \left.+2 k\left(x_{4}, x_{3}, y_{3}\right)\right]+O\left(h^{5}\right) .
\end{aligned}
$$

An approximate value of $y$ at $x_{0}+5 h$ is obtained by the open Newton-Cotes formula

$$
\begin{aligned}
y\left(x_{0}+5 h\right)=f\left(x_{0}+5 h\right)+\frac{5 h}{24}\left[11 k\left(x_{5}, x_{1}, y_{1}\right)+k\left(x_{5}, x_{2}, y_{2}\right)\right. \\
\left.\quad+k\left(x_{5}, x_{3}, y_{3}\right)+11 k\left(x_{5}, x_{4}, y_{4}\right)\right]+O\left(h^{5}\right)
\end{aligned}
$$

combined with the closed Newton-Cotes formulae

$$
\begin{aligned}
y\left(x_{0}+5 h\right)=f\left(x_{0}+5 h\right)+\frac{5 h}{288}[ & 19 k\left(x_{5}, x_{0}, y_{0}\right)+75 k\left(x_{5}, x_{1}, y_{1}\right) \\
& +50 k\left(x_{5}, x_{2}, y_{2}\right)+50 k\left(x_{5}, x_{3}, y_{3}\right) \\
& \left.+75 k\left(x_{5}, x_{4}, y_{4}\right)+19 k\left(x_{5}, x_{5}, y_{5}\right)\right]+O\left(h^{6}\right) .
\end{aligned}
$$

To advance from $x_{0}+5 h$ to $x_{0}+6 h$ we use the open Newton-Cotes formula

$$
\begin{aligned}
y_{6}=f\left(x_{0}+6 h\right) & +\frac{6 h}{20}\left[11 k\left(x_{0}+6 h, x_{1}, y_{1}\right)-14 k\left(x_{6}, x_{2}, y_{2}\right)\right. \\
& \left.+26 k\left(x_{6}, x_{3}, y_{3}\right)-14 k\left(x_{6}, x_{4}, y_{4}\right)+11 k\left(x_{6}, x_{5}, y_{5}\right)\right]+O\left(h^{7}\right)
\end{aligned}
$$

together with Weddle's rule

$$
\begin{aligned}
y_{6}= & f\left(x_{0}+6 h\right)+\frac{3 h}{10}\left[k\left(x_{6}, x_{0}, y_{0}\right)+5 k\left(x_{6}, x_{1}, y_{1}\right)+k\left(x_{6}, x_{2}, y_{2}\right)\right. \\
& \left.+6 k\left(x_{6}, x_{3}, y_{3}\right)+k\left(x_{6}, x_{4}, y_{4}\right)+5 k\left(x_{6}, x_{5}, y_{5}\right)+k\left(x_{6}, x_{6}, y_{6}\right)\right]+O\left(h^{7}\right) .
\end{aligned}
$$

The Newton-Cotes open and closed formulae and Weddle's rule are given in Milne [7]. For the other integration rules used here, see Hildebrand [3]. It should be noted that we have assumed that the eighth partial derivative of $k$ with respect to $s$ and $y(s)$ exist and is bounded in order to apply our method.

The method under consideration can be applied to systems of integral equations.

4. Use of Gregory-Newton Formulae. The Gregory-Newton Formulae (see Todd [11], Hildebrand [3])

$$
\begin{aligned}
& \int_{x_{0}}^{x_{0}+n h} f(p) d p=h\left\{\frac{f\left(x_{0}\right)}{2}+f\left(x_{1}\right)+\cdots+f\left(x_{n-1}\right)+\frac{f\left(x_{n}\right)}{2}\right\} \\
& +\frac{h}{12}\left\{\left[f\left(x_{1}\right)-f\left(x_{0}\right)\right]-\left[f\left(x_{n}\right)-f\left(x_{n-1}\right)\right]\right\} \\
& \quad-\frac{h}{24}\left\{\left[f\left(x_{2}\right)-2 f\left(x_{1}\right)+f\left(x_{0}\right)\right]+\left[f\left(x_{n}\right)-2 f\left(x_{n-1}\right)+f\left(x_{n-2}\right)\right]\right\}
\end{aligned}
$$




$$
\begin{aligned}
& +\frac{19 h}{720}\left\{\left[f\left(x_{3}\right)-3 f\left(x_{2}\right)+3 f\left(x_{1}\right)-f\left(x_{0}\right)\right]-\left[f\left(x_{n}\right)-3 f\left(x_{n-1}\right)\right.\right. \\
& \left.\left.+3 f\left(x_{n-2}\right)-f\left(x_{n-3}\right)\right]\right\} \\
& -\frac{3 h}{160}\left\{\left[f\left(x_{4}\right)-4 f\left(x_{3}\right)+6 f\left(x_{2}\right)-4 f\left(x_{1}\right)+f\left(x_{0}\right)\right]\right. \\
& \left.\quad+\left[f\left(x_{n}\right)-4 f\left(x_{n-1}\right)+6 f\left(x_{n-2}\right)-4 f\left(x_{n-3}\right)+f\left(x_{n-4}\right)\right]\right\} \\
& +\frac{863 h}{60480}\left[\Delta^{5} f\left(x_{0}\right)-\nabla^{5} f\left(x_{n}\right)\right]+\cdots
\end{aligned}
$$

was used by Fox and Goodwin [2] in their treatment of linear Volterra integral equations. In this paper we use the Gregory-Newton formulae through fourth differences to advance the solution from $x=x_{0}+6 h$ to any $x=x_{0}+N h$.

Since the integral equation is nonlinear, there is a need for a "predictor" to correspond to the role of the Gregory-Newton formula as "corrector." In our work we have used the following scheme. If we are to advance from $x_{0}+(2 N-1) h$ to $x_{0}+2 N h$ use Simpson's rule with step size $h$, from $x_{0}$ through $x_{0}+2 N h-4 h$, then use the open Newton-Cotes formulae

$$
\int_{x_{0}}^{x_{4}} y d x=\frac{4 h}{3}\left[2 y_{1}-y_{2}+2 y_{3}\right]+O\left(h^{5}\right)
$$

on the interval $\left[x_{0}+2 N h-4 h, x_{0}+2 N h\right]$. In case $x=x_{0}+(2 N-1) h$ we first integrate from $x_{0}$ to $x_{0}+3 h$ with Simpson's "three-eighths" rule followed by Simpson's rule until we come to $x_{0}+(2 N-1) h-4 h$. Then apply the open Cotes formula used above. This predictor has enabled us to use the Gregory-Newton formula with only two iterations. Before using this, an $O\left(h^{2}\right)$ predictor was user. However seven iterations were necessary in this case. Here the iterations wre. stopped after a certain number of decimal places of accuracy were achieved.

5. Computational Examples. The following computational examples were com puted in Fortran (single precision) on the CDC 1604. By error we mean

$$
\text { error }=\mid \text { true }- \text { approximate value } \mid \text {. }
$$

Example 1. The integral equation

$$
y(t)=1-t+\int_{0}^{t}\left(t e^{x(t-2 x)}+e^{-2 x^{2}}\right) \cdot(y(x))^{2} d x
$$

has the solution $y(x)=e^{x^{2}}$. It has been considered by Laudet and Oules [6]. W. find the following errors.

Example 2. The integral equation

$$
y(t)=\frac{2 t^{3 / 2}}{3}+\int_{0}^{t}(y(x))^{1 / 2} d x
$$

was obtained by integrating the differential equation $y^{\prime}=x^{1 / 2}+y^{1 / 2}, y(0)=-1$. This differential equation (see Todd [11], Noble [9]) does not possess a Tay':.... 
pansion about the origin. Its solution about the origin can be written in the series

$$
y(x)=\frac{2}{3} x^{3 / 2}+\frac{4}{7}(2 / 3)^{1 / 2} x^{7 / 4}+\frac{1}{7} x^{2}+\frac{1}{49}(2 / 3)^{1 / 2} x^{9 / 4}-\frac{2}{1715} x^{5 / 2}+\cdots
$$

we obtain the following values for $x$ at $.1, .2,1.0$ with step sizes $.1, .05, .025$.

These values compare quite favorably with those obtained by Noble using the Runge-Kutta method (see Noble [9]).

Example 3. The integral equation

$$
y(t)=\int_{0}^{t} \max (x, y) d x
$$

was obtained from the differential equation $y^{\prime}=\max (x, y), y(0)=0$ (see Burkill [1]). The solution of this differential equation is

$$
y(x)=x^{2} / 2 \text { for } x \leqq 2, \quad y(x)=2 e^{(x-2)} \text { for } x>2 .
$$

Thus there is a discontinuity in $y^{\prime \prime}$ at $x=2$.

In this example somewhat better results in the region $x \geqq 2$ were obtained by using the Runge-Kutta method.

Example 4. The integral equation

$$
y(t)=2 t+3+\int_{0}^{t}-y(x)(2(t-x)+3) d x
$$

discussed by Todd [11]. The equation has the exact solution $y(t)=4 e^{-2 t}-e^{-t}$.

In addition to the above examples the writer has computed examples given by Jones [4], Pouzet [10], Fox and Goodwin [2] and others. These numerical examples are available from the writer in an MRC report.

TABLE 1

\begin{tabular}{l|c|c|c}
\hline$x$ & $h=.05$ & $h=.1$ & $h=.2$ \\
\hline .05 & $2.91 \times 10^{-11}$ & & \\
.1 & 0 & $2.91 \times 10^{-10}$ & \\
.2 & 0 & $2.65 \times 10^{-9}$ & $4.94 \times 10^{-8}$ \\
.25 & $2.91 \times 10^{-11}$ & & \\
.3 & $2.91 \times 10^{-11}$ & $3.84 \times 10^{-9}$ & \\
.5 & 0 & $2.35 \times 10^{-8}$ & \\
1.00 & $2.33 \times 10^{-10}$ & $2.40 \times 10^{-8}$ & $7.65 \times 10^{-5}$ \\
2.00 & $1.80 \times 10^{-6}$ & $9.07 \times 10^{-5}$ & $3.51 \times 10^{-3}$ \\
2.50 & $1.15 \times 10^{-4}$ & $5.79 \times 10^{-3}$ & \\
\hline
\end{tabular}

TABLE 2

\begin{tabular}{l|c|c|c}
\hline & $h=.1$ & $h=.05$ & $h=.025$ \\
\hline$x=.1$ & .030711 & .030838 & .030860 \\
$x=.2$ & .093425 & .093541 & .093621 \\
$x=1$ & 1.290677 & 1.291174 & 1.291354 \\
\hline
\end{tabular}


TABLE 3

\begin{tabular}{c|c|c|c}
\hline$x$ & $h=.05$ & $h=.1$ & $h=.2$ \\
\cline { 2 - 3 } .1 & $1.14 \times 10^{-13}$ & $1.14 \times 10^{-13}$ & \\
.2 & $1.36 \times 10^{-12}$ & $4.55 \times 10^{-13}$ & $4.55 \times 10^{-13}$ \\
.3 & $9.09 \times 10^{-31}$ & 0 (Machine) & \\
.4 & $3.64 \times 10^{-12}$ & $5.46 \times 10^{-12}$ & $1.82 \times 10^{-12}$ \\
.5 & 0 (Machine) & 0 (Machine) & \\
1.0 & 0 (Machine) & 0 (Machine) & 0 (Machine) \\
1.4 & $2.91 \times 10^{-11}$ & $1.46 \times 10^{-11}$ & $1.46 \times 10^{-11}$ \\
1.6 & $8.73 \times 10^{-11}$ & $5.82 \times 10^{-11}$ & $5.82 \times 10^{-11}$ \\
1.8 & $5.82 \times 10^{-11}$ & $5.82 \times 10^{-11}$ & $8.73 \times 10^{-11}$ \\
2.0 & $0(\mathrm{Machine})$ & $0(\mathrm{Machine})$ & $1.04 \times 10^{-9}$ \\
2.1 & $8.19 \times 10^{-5}$ & $1.76 \times 10^{-3}$ & \\
2.2 & $2.58 \times 10^{-4}$ & $4.70 \times 10^{-4}$ & $7.27 \times 10^{-3}$ \\
2.5 & $3.43 \times 10^{-4}$ & $1.36 \times 10^{-3}$ & \\
3.0 & & $2.26 \times 10^{-3}$ & $8.99 \times 10^{-3}$ \\
\hline
\end{tabular}

TABLE 4

\begin{tabular}{c|c|c|c}
\hline$x$ & $h=.05$ & $h=.1$ & $h=.2$ \\
\hline & $2.41 \times 10^{-6}$ & $2.44 \times 10^{-5}$ & \\
.1 & $2.48 \times 10^{-6}$ & $6.83 \times 10^{-5}$ & $7.36 \times 10^{-4}$ \\
.3 & $1.38 \times 10^{-7}$ & $4.17 \times 10^{-5}$ & \\
.4 & $1.22 \times 10^{-7}$ & $1.33 \times 10^{-4}$ & $1.68 \times 10^{-3}$ \\
.5 & $2.98 \times 10^{-8}$ & $1.87 \times 10^{-4}$ & \\
1.0 & $8.57 \times 10^{-9}$ & $1.65 \times 10^{-5}$ & $6.66 \times 10^{-3}$ \\
1.4 & $1.49 \times 10^{-9}$ & $4.62 \times 10^{-6}$ & $8.83 \times 10^{-4}$ \\
1.6 & $6.43 \times 10^{-9}$ & $2.37 \times 10^{-6}$ & $3.34 \times 10^{-3}$ \\
1.8 & $9.70 \times 10^{-9}$ & $1.14 \times 10^{-6}$ & $4.63 \times 10^{-4}$ \\
2.0 & $1.25 \times 10^{-8}$ & $4.69 \times 10^{-7}$ & $1.35 \times 10^{-3}$ \\
2.5 & $1.35 \times 10^{-8}$ & $8.57 \times 10^{-8}$ & \\
3.0 & & $1.20 \times 10^{-7}$ & $3.45 \times 10^{-4}$ \\
4.0 & & $3.70 \times 10^{-8}$ & $4.65 \times 10^{-6}$ \\
5.0 & & $8.94 \times 10^{-8}$ & $4.43 \times 10^{-5}$ \\
\hline
\end{tabular}

5. Acknowledgements. The writer expresses his appreciation to Professor B. Noble for many helpful comments and suggestions while writing the paper. The above work was performed at the Mathematics Research Center, U. S. Army, under Contract No.: DA-11-022-ORD-2059.

\footnotetext{
Mathematics Research Center, United States Army

University of Wisconsin

Madison, Wisconsin
}

1. J. C. Burkill, The Theory of Ordinary Differential Equations, Oliver and Boyd, New York, 1962, pp. 11, 109.

2. L. Fox \& E. T. Goodwin, "The numerical solution of nonsingular linear integral equations," Philos. Trans. Roy. Soc. London Ser. A., v. 245, 1953, pp. 501-534. MR 14, 908.

3. F. B. Hildebrand, Introduction to Numerical Analysis, McGraw-Hill, New York, 1956. MR 17, 788 .

4. J. G. Jones, "On the numerical solution of convolution integral equations and systems of such equations," Math. Comp., v. 15, 1961, pp. 131-142. MR 22 \$12728. 
5. J.Kuntzmann, "Neuere Entwicklungen der Methode von Runge und Kutta," $Z$. Angew. Math. Mech., v. 41, 1961, pp. 28-31.

6. M. LAUdeT \& H. Oules, "Sur l'intégration numérique des équations intégrales du type Volterra," Symposium on the Numerical Treatment of Ordinary Differential Equations, Integral and Integro-differential Equations, pp. 117-121, Birkhäuser Verlag, Basel, 1960. MR 23 * B597.

7. W. E. Minne, Numerical Calculus, Princeton Univ. Press, Princeton, N. J., 1949, pp. 123-127. MR 10, 483 .

8. B. NOBLE, "The numerical solution of nonlinear integral equations and related topics," Nonlinear Integral Equations, Univ. of Wisconsin Press, Madison, Wis., 1964, pp. 215-318. MR 30 * 3582 .

9. B. Noble, Numerical Methods, Vol. 2: Differences, Integration and Differential Equations, Oliver and Boyd, New York, 1964, pp. 267, 330.

10. P. Pouzet, "Mèthode d'intégration numérique des équations intégrales et intégrodifférentielles du type de Volterra de seconde espèce. Formules de Runge Kutta," Symposium on the Numerical Treatment of Ordinary Differential Equations, Integral and Integro-differential Equations, pp. 362-368, Birkhäuser Verlag, Basel, 1960. MR 23 * B601.

11. J. TodD Editor "Classical numerical analysis," Survey of Numerical Analysis, edited by J. Todd, McGraw-Hill, New York, 1962, pp. 72-73, 80. MR 24 *B1271. 